\title{
Epidemiología de la prematuridad y sus determinantes, en una población de mujeres adolescentes y adultas de Colombia
}

\author{
Luis Alfonso Mendoza Tascón ${ }^{1,2}$, Diana Isabel Claros Benítez ${ }^{2}$, Miguel Ángel Osorio \\ Ruíz ${ }^{3}$, Laura Isabel Mendoza Tascón ${ }^{2}$, Claudia Bibiana Peñaranda Ospina ${ }^{1,2}$, Jorge \\ Hernán Carrillo Bejarano ${ }^{1}$, Fernando Londoño Romero. ${ }^{1}$
}

${ }^{1}$ Fundación Hospital San José de Buga. Colombia. ${ }^{2}$ Facultad Ciencias de la Salud, Programa de Medicina, Unidad Central del Valle del Cauca, Tuluá, Colombia. ${ }^{3}$ Departamento de Pediatría, Universidad del Valle, Cali, Colombia.

\section{RESUMEN}

Antecedentes: La prematuridad es considerada actualmente una prioridad en salud pública para algunos países. Objetivo: Describir la prevalencia del parto prematuro y sus determinantes, en una población de gestantes adolescentes y adultas, que tuvieron su único o último parto en la Fundación Hospital San José de Buga, Colombia, entre los años 2010 a 2015. Métodos. Estudio de casos y controles realizado con 11.881 neonatos mujeres adolescentes y adultas. Los datos fueron analizados en Stata $\otimes 11.0$, empleando para variables cuantitativas, mediana o promedio y sus medidas de dispersión, comparadas mediante pruebas t, Ranksum, ANOVA o Kruskal Wallis, de acuerdo a su distribución y para variables categóricas; medidas de frecuencia absolutas y relativas, comparadas mediante Pruebas de Chi2 o exacta de Fisher. Para estimar las asociaciones empleamos Odds Ratio con sus intervalos de confianza de $95 \%$ y para el análisis multivariado la regresión logística. La significancia estadística fue definida como un valor $p<0,05$. Resultados. La prevalencia de prematuridad fue 11,4\%. Después del análisis multivariado, los determinantes asociados a prematuridad fueron etnia indígena-afrocolombiana o mulato, número de controles prenatales igual o inferior a 6, periodo intergenésico inferior a 2 años, embarazo múltiple, preeclampsia, oligohidramnios, restricción del crecimiento intrauterino y hemorragia del tercer trimestre (incluye desprendimiento prematuro de placenta). Conclusiones. La prematuridad es el resultado de una compleja red de determinantes individuales, sociales, culturales y gestacionales que interactúan, por lo que para su prevención se debe no sólo trabajar en medidas de salud, sino en elaboración de políticas y planes de acción integral.

\section{PALABRAS CLAVE: Prematuridad, determinantes de la salud, mujer adolescente, mujer adulta}

\section{SUMMARY}

Background: Prematurity is currently considered a public health priority for some countries. Objective: To describe the prevalence of preterm birth and its determinants in a population of adolescent and adult mothers who had their sole or last delivery in the Fundación Hospital San José de Buga, Colombia, between 2010-2015. Methods: Case-control study conducted with 11,881 infants adolescent and adult women. The data were analyzed in Stata 11.0, using quantitative, medium or average and dispersion measures variables compared using $t$ tests, Ranksum, ANOVA or Kruskal Wallis, according to distribution and for categorical variables, frequency measurements absolute and relative, compared with Chi2 tests or Fisher exact. To estimate associations employ odds ratio with confidence intervals of $95 \%$ and multivariate analysis logistic regression. Statistical significance was defined as $p<0.05$. Results: Prematurity prevalence was $11.4 \%$. 
After multivariate analysis, the determinants associated with prematurity were indigenous-Afro-Colombian or mulatto ethnicity, number of prenatal visits equal to or less than 6, less than two years intergenesic period, multiple pregnancy, preeclampsia, oligohydramnios, IUGR and bleeding of the third quarter (includes abruption). Conclusions: Prematurity is the result of a complex network of individual, social, cultural and gestational determinants that interact, so that prevention is due not only work on health measures, but in developing policies and plans integral action.

\section{KEY WORDS: Prematurity, determinants of health, adolescent female, adult woman}

\section{INTRODUCCIÓN}

Los determinantes sociales y ambientales, tienen dos veces el impacto en la calidad de la atención en salud y en la salud en general de un individuo, que los otros determinantes (1). Mientras varios de ellos afectan el embarazo en mujeres adolescentes y adultas como: pobreza, estructura familiar, calidad de la vivienda, acceso a alimentos saludables, acceso a servicios de salud y de atención primaria, tecnología en salud, cohesión social, percepción de discriminación/inequidad, acceso a empleo, situación laboral, políticas que apoyen la promoción de la salud, entornos escolares seguros e ingreso a la educación superior (2).

La prematuridad que representa $10-12 \%$ de todos los nacimientos, es resultado de varios determinantes de la salud, convirtiéndose también en un determinante importante de mortalidad, morbilidad neonatal, complicaciones y altos costos de tratamiento (3-10), siendo concebida por algunos países, como de alta prioridad para la salud pública. La prematuridad es causa importante de mortalidad, donde un $28 \%$ de las muertes están asociadas a esta (11), siendo para algunos países, la principal causa de muerte infantil (12). Un $75-80 \%$ de muertes perinatales ocurren en $<37$ semanas de edad gestacional (13-15) y aproximadamente $40 \%$ ocurren en $<32$ semanas (16). Las complicaciones secundarias al parto prematuro, son la causa directa más importante de mortalidad neonatal, siendo responsable de $35 \%$ del total de las muertes a nivel mundial, con 3,1 millones de muertes al año y la segunda causa de muerte más común en $<5$ años después de la neumonía (12).

El objetivo de este estudio es describir la prevalencia del parto prematuro y evaluar sus determinantes asociados, en una población de gestantes adolescentes y adultas, que tuvieron su único o último parto en la Fundación Hospital San José de Buga, Colombia entre los años 2010 a 2015.

\section{MATERIALES Y MÉTODOS}

Diseño del estudio. La información para esta investigación fue recolectada como parte de un estudio analítico de tipo casos y controles.
Contexto. Este trabajo se realizó en la ciudad de Buga, Colombia, en la Fundación Hospital San José de Buga.

Población de estudio. La población estuvo constituida por mujeres adolescentes (10-19 años) y mujeres adultas ( $\geq 20$ años), quienes tuvieron su primer o último parto en esta institución de salud, entre los años 2010 y 2015. Definimos caso, a los nacimientos que ocurrieron antes de completarse las 37 semanas o antes de 259 días de gestación, desde el primer día del último periodo menstrual $(17,18)$, y controles a los neonatos nacidos $\geq 37$ semanas de gestación o $\geq 259$ días.

Criterios de inclusión. Neonatos y sus madres adolescentes de 10-19 años y adultas $\geq 20$ años de edad al momento del parto, procedentes de Buga y otros lugares del Valle del Cauca u otros municipios del país, cuyo único o último hijo haya nacido en la Fundación Hospital San José de Buga, entre los años 2010 y 2015.

Criterios de exclusión. Neonatos y madres adolescentes de 10-19 años y adultas $\geq 20$ años de edad al momento del parto, con registros incompletos.

\section{Variables}

Dependientes. Prematuridad: el parto prematuro es definido como el nacimiento que ocurre antes de completarse las 37 semanas de gestación o antes de 259 días de gestación, desde el primer día del último periodo menstrual o por una ecografía temprana o por la Clasificación de Ballard Modificado.

Independientes. Determinantes maternos: edad materna, procedencia, área de residencia (urbana o rural), número de embarazos, número de nacidos vivos. Determinantes de la gestación: número de controles prenatales, tipo de parto, período intergenésico. Determinantes sociales, culturales y educativos: educación materna, etnia, seguridad social en salud, estado civil materno.

Fuente de los datos. Los datos fueron obtenidos de base de datos que se lleva en la Fundación Hospital San José de Buga, desde 01-Enero-2010 a 31 -Diciembre-2015 y cuyos objetivos son investigativos. 
Método de selección de los participantes. Se incluyeron todos los participantes que cumplieron con los criterios de inclusión y no tenían criterios de exclusión.

Limitaciones y sesgos. Los principales sesgos tenidos en cuenta fueron los de información, controlándose mediante la exclusión de aquellas participantes que no tuvieran la información completa requerida en la investigación y que esta fuera relevante.

Tamaño de la muestra y muestreo. El tamaño de la muestra estuvo constituida por 3.003 mujeres adolescentes y 8.878 mujeres adultas. Dado que se trabajó con la totalidad de la población que cumplió con los criterios, no se realizó muestreo alguno para la selección de los participantes.

Análisis estadístico. Los datos fueron analizados en Stata ${ }^{\circledR}$ 11.0. Se emplearon frecuencias absolutas y relativas, promedio y desviación estándar, mediana y rango intercuartíl, comparadas mediante Pruebas Chi2 o exacta de Fisher, t de Student, Ranksum, ANOVA o Kruskal Wallis, según correspondiera y de acuerdo a su distribución. Para estimar las asociaciones empleamos odds ratio (OR) con su intervalo de confianza de 95\% (IC 95\%). En el análisis multivariado se empleó la regresión logística. La significancia estadística fue definida como un valor $\mathrm{p}<0,05$.

Esta investigación fue aprobado por los Comité de Ética de la Fundación Hospital San José de Buga y de la Facultad de Ciencias de la Salud, de la Unidad Central del Valle del Cauca, Tuluá, Colombia, y carece de conflicto de intereses desde el punto de vista de patrocinios o remuneración.

\section{RESULTADOS}

Entre los años 2010-2015 nacieron, 11.881 neonatos, entre ellos 1.352 prematuros $(11,4 \%)$. Entre las adolescentes (n: 3.003 ) hubo 346 (25,6\%), entre las de $20-35$ años $890(65,8 \%)$ y entre las $\geq 36$ años 112 (8,3\%) nacimientos prematuros, diferencia que fue significativa $(p<0,0010)$. Entre los prematuros, $34(2,5 \%)$ fueron $<28,111(8,2 \%)$ de $28-31$ y $1.207(89,3 \%)$ de $32-36$ semanas gestacionales (Tabla I). Los prematuros tardíos (3436 semanas), correspondieron a 1.060 nacimientos $(78,4 \%)$.

Entre los determinantes evaluados para parto de término y prematuro, encontramos diferencias significativa para número de embarazos, número de hijos nacidos vivos, escolaridad materna, etnia afrocolombiana y mulata, otras etnias, carencia de seguridad social en salud, número de controles prenatales, periodo intergenésico, embarazo múltiple, preeclampsia, ruptura prematura de membranas, corioamnionitis, oligohidramnios, hipertensión arterial crónica, diabetes gestacional, obesidad, vaginosis y hemorragias del tercer trimestre, incluyendo desprendimiento prematuro de placenta. No se halló diferencia significativa entre parto a término y pretermino para determinantes como, edad materna, procedencia rural o urbana, carencia de esposo o compañero estable, infección de vías urinarias y polihidramnios (Tabla II).

Cuando comparamos la presencia de estos determinantes entre las mujeres adultas ( $\geq 20$ años) y las adolescentes (<20 años), encontramos diferencia significativas para edad materna, procedencia rural, número de embarazos e hijos nacidos vivos, escolaridad materna, carencia de seguridad social en salud, carencia de esposo o compañero estable, número de controles prenatales, periodo intergenésico, embarazo múltiple, preeclampsia, corioamnionitis, infección de vías urinarias, hipertensión arterial crónica, diabetes gestacional, polihidramnios, obesidad y vaginosis. No hallamos diferencia significativa entre nacidos a término y prematuros, para etnia, ruptura prematura de membranas, oligohidramnios, restricción del crecimiento intrauterino y hemorragia del tercer trimestre (incluye desprendimiento prematuro) (Tabla II).

Tabla I

PREVALENCIA DE LA PREMATURIDAD SEGÚN GRUPOS DE EDAD

\begin{tabular}{|c|c|c|c|c|c|c|c|c|}
\hline \multirow[t]{2}{*}{ Edad gestacional } & \multicolumn{2}{|c|}{$\begin{array}{c}\text { Madres de } \\
10 \text { a } 19 \text { años }\end{array}$} & \multicolumn{2}{|c|}{$\begin{array}{c}\text { Madres de } \\
20 \text { a } 35 \text { años }\end{array}$} & \multicolumn{2}{|c|}{$\begin{array}{c}\text { Madres de } \\
36 \text { y más años }\end{array}$} & \multirow{2}{*}{$\begin{array}{c}\text { Total } \\
\mathrm{n}\end{array}$} & \multirow[b]{2}{*}{$\%$} \\
\hline & $\mathrm{n}$ & $\%$ & $\mathrm{n}$ & $\%$ & $\mathrm{n}$ & $\%$ & & \\
\hline$<28$ semanas & 8 & 0,6 & 24 & 1,8 & 2 & 0,2 & 34 & 2,5 \\
\hline 28-31 semanas & 30 & 2,2 & 68 & 5,0 & 13 & 1,0 & 111 & 8,2 \\
\hline 32-36 semanas & 308 & 22,8 & 802 & 59,0 & 97 & 7,1 & 1207 & 89,3 \\
\hline Total $<37$ semanas & 346 & 25,6 & 890 & 65,8 & 112 & 8,3 & 1352 & 100,0 \\
\hline
\end{tabular}




\section{DETERMINANTES DEL PARTO A TÉRMINO Y PREMATURO}

\begin{tabular}{|c|c|c|c|}
\hline Determinante & $\begin{array}{l}\text { Neonato a } \\
\text { término } \\
\mathrm{n}: 10529\end{array}$ & $\begin{array}{l}\text { Neonato } \\
\text { prematuro } \\
\text { n: } 1352\end{array}$ & Valor $\mathrm{p}$ \\
\hline \multicolumn{4}{|l|}{ Maternos } \\
\hline Edad (años) & 23 (19-29) & $23(19-29)$ & 0,9635 \\
\hline Procedencia rural & $2400(22,8 \%)$ & $301(22,3 \%)$ & 0,6862 \\
\hline $\mathrm{N}^{\circ}$ de embarazos & $1(1-2)$ & $2(1-3)$ & $<0,0001$ \\
\hline$N^{\circ}$ de nacidos vivos & $1(1-2)$ & $2(1-2)$ & $<0,0001$ \\
\hline \multicolumn{4}{|l|}{ Sociales, culturales y educativos } \\
\hline Escolaridad (años) & $9(5-11)$ & $9(7-11)$ & 0,0164 \\
\hline Etnia indígena & $9(0,1 \%)$ & $3(0,2 \%)$ & 0,3022 \\
\hline Etnia afrocolombiano; mulato & $2854(27,1 \%)$ & $433(32 \%)$ & 0,0002 \\
\hline Otras etnias & $7666(72,8 \%)$ & $916(67,8 \%)$ & 0,0001 \\
\hline Carencia de seguridad social en salud & $539(51 \%)$ & $813(60,1 \%)$ & $<0,0001$ \\
\hline Sin esposo o compañero & $1628(15,5)$ & $236(17,5 \%)$ & 0,0632 \\
\hline \multicolumn{4}{|l|}{ Gestacionales } \\
\hline $\mathrm{N}^{\circ}$ de CPN & $8(7-9)$ & $6(5-7)$ & $<0,0001$ \\
\hline Período intergenésico (meses) & $59(33-100)$ & $47(23-91)$ & $<0,0001$ \\
\hline Embarazo múltiple & $68(0,6 \%)$ & $147(10,9 \%)$ & $<0,0001$ \\
\hline Preeclampsia & $769(7,3 \%)$ & $284(21 \%)$ & $<0,0001$ \\
\hline Ruptura prematura de membranas & $1084(10,3 \%)$ & $168(12,4 \%)$ & 0,0185 \\
\hline Corioamnionitis & $183(1,7 \%)$ & $60(4,4)$ & $<0,0001$ \\
\hline Oligohidramnios & $152(1,4 \%)$ & $78(5,8 \%)$ & $<0,0001$ \\
\hline Infección urinaria & $1585(15,1 \%)$ & $186(13,8 \%)$ & 0,2227 \\
\hline Hipertensión arterial crónica & $359(3,4 \%)$ & $63(4,6 \%)$ & 0,0238 \\
\hline Diabetes gestacional & $1410(13,4 \%)$ & $102(7,5 \%)$ & $<0,0001$ \\
\hline Restricción del crecimiento intrauterino & $553(5,3 \%)$ & $140(10,4 \%)$ & $<0,0001$ \\
\hline Polihidramnios & $194(1,8 \%)$ & $24(1,8 \%)$ & 0,9473 \\
\hline Obesidad & $1631(15,5 \%)$ & $66(4,9 \%)$ & $<0,0001$ \\
\hline Vaginosis & $2653(25,2 \%)$ & $113(8,4 \%)$ & $<0,0001$ \\
\hline Hemorragia del 3er trimestre (incluyendo DPP) & $27(0,26 \%)$ & $54(4 \%)$ & $<0,0001$ \\
\hline
\end{tabular}

DPP: Desprendimiento prematuro de placenta

Entre el grupo de adolescentes fue significativamente más frecuente la procedencia rural, un menor número de embarazos e hijos nacidos vivos, carencia de seguridad social en salud, carencia de esposo o compañero estable, menor número de controles prenatales, periodo intergenésico más corto, corioamnionitis, infección de vías urinarias y vaginosis. Mientras que en el grupo de mujeres adultas fue significativamente más frecuente el embarazo múltiple, preeclampsia, hipertensión arterial crónica, diabetes gestacional, polihidramnios y obesidad (Tabla III).

En el análisis bivariado, hallamos asociación significativa con prematuridad para etnia indígenaafrocolombiana o mulato, carencia de seguridad social en salud, número de embarazos $\geq 2$, número de controles prenatales igual o inferior a 6 , periodo intergenésico inferior a 2 años, embarazo múltiple, preeclampsia, ruptura prematura de membranas, corioamnionitis, oligohidramnios, hipertensión arterial crónica, restricción del crecimiento intrauterino y hemorragia del tercer trimestre (incluye desprendimiento prematuro de placenta). En el análisis multivariado se encontró asociado a prematuridad la etnia indígena-afrocolombiana o mulato, número de controles prenatales igual o inferior a 6 , periodo intergenésico inferior a 2 años, embarazo múltiple, preeclampsia, oligohidramnios, restricción del crecimiento intrauterino y hemorragia del tercer trimestre (incluye desprendimiento prematuro de placenta) (Tabla IV). 


\section{DETERMINANTES ENTRE MUJERES ADULTAS Y ADOLESCENTES}

\begin{tabular}{|c|c|c|c|}
\hline Determinante & $\begin{array}{c}\text { Adulta } \\
\mathrm{n}: 8878\end{array}$ & $\begin{array}{c}\text { Adolescente } \\
\text { n: } 3003\end{array}$ & Valor $\mathrm{p}$ \\
\hline \multicolumn{4}{|l|}{ Maternos } \\
\hline Edad (años) & $26(22-31)$ & $17(16-19)$ & $<0,0001$ \\
\hline Procedencia rural & $1878(21,2 \%)$ & $858(28,6 \%)$ & $<0,0001$ \\
\hline $\mathrm{N}^{\circ}$ de embarazos & $2(1-3)$ & $1(1-1)$ & $<0,0001$ \\
\hline $\mathrm{N}^{\circ}$ de nacidos vivos & $2(1-2)$ & $1(1-1)$ & $<0,0001$ \\
\hline \multicolumn{4}{|l|}{ Sociales, culturales y educativos } \\
\hline Escolaridad (años) & $9(5-11)$ & $9(7-11)$ & 0,0255 \\
\hline Etnia indígena & $8(0,09 \%)$ & $3(0,1 \%)$ & 0,8457 \\
\hline Etnia afrocolombiano; mulato & $2489(20 \%)$ & $830(26,6 \%)$ & 0,6927 \\
\hline Otras etnias & $6381(71,9 \%)$ & $2170(72,3 \%)$ & 0,7007 \\
\hline Carencia de seguridad social en salud & $4238(47,7 \%)$ & $1943(64,7 \%)$ & $<0,0001$ \\
\hline Sin esposo o compañero & $1098(12,4 \%)$ & $803(26,7 \%)$ & $<0,0001$ \\
\hline \multicolumn{4}{|l|}{ Gestacionales } \\
\hline $\mathrm{N}^{\circ}$ de CPN & $8(7-9)$ & $7(6-8)$ & $<0,0001$ \\
\hline Período intergenésico (meses) & $62(35-103)$ & $27(18-35)$ & $<0,0001$ \\
\hline Embarazo múltiple & $184(2,1 \%)$ & $37(1,2 \%)$ & 0,0041 \\
\hline Preeclampsia & $1161(13 \%)$ & $273(9,1 \%)$ & $<0,0001$ \\
\hline Ruptura prematura de membranas & $961(10,8 \%)$ & $332(11,1 \%)$ & 0,7508 \\
\hline Corioamnionitis & $210(2,4 \%)$ & $109(3,6 \%)$ & 0,0003 \\
\hline Oligohidramnios & $252(2,8 \%)$ & $99(3,3 \%)$ & 0,2226 \\
\hline Infección urinaria & $1235(13,9 \%)$ & $501(16,7 \%)$ & 0,0002 \\
\hline Hipertensión arterial crónica & $475(5,4 \%)$ & $14(0,47 \%)$ & $<0,0001$ \\
\hline Diabetes gestacional & $1094(12,3 \%)$ & $126(4,2 \%)$ & $<0,0001$ \\
\hline Restricción del crecimiento intrauterino & $734(8,3 \%)$ & $225(7,5 \%)$ & 0,1905 \\
\hline Polihidramnios & $240(2,7 \%)$ & $14(0,47 \%)$ & $<0,0001$ \\
\hline Obesidad & $950(10,7 \%)$ & $211(7 \%)$ & $<0,0001$ \\
\hline Vaginosis & $1238(13,9 \%)$ & $674(22,4 \%)$ & $<0,0001$ \\
\hline Hemorragia del 3er (incluyendo DPP) & $216(2,4 \%)$ & $56(1,9 \%)$ & 0,0838 \\
\hline
\end{tabular}

CPN: Control prenatal. DPP: Desprendimiento prematuro de placenta.

Tabla IV

ASOCIACIÓN ENTRE DETERMINANTES Y PREMATURIDAD

\begin{tabular}{lrrrr}
\hline Determinante & ORc & Valor $p$ & ORa & Valor $p$ \\
\hline Etnia indígena, afrocolombiano o mulato & $1,25(1,10-1,41)$ & $<0,0001$ & $1,22(1,01-1,47$ & 0,0310 \\
Carencia de seguridad social en salud & $1,45(1,29-1,63)$ & $<0,0001$ & $1,12(0,94-1,33)$ & 0,2110 \\
$\mathrm{~N}^{\circ}$ de embarazos $\geq 2$ & $1,34(1,17-1,53)$ & $<0,0001$ & $1,12(0,94-1,33)$ & 0,2110 \\
$\mathrm{~N}^{\circ}$ de CPN 0-6 & $3,60(3,20-4,05)$ & $<0,0001$ & $3,13(2,62-3,73)$ & $<0,0001$ \\
Período intergenésico $<2$ años & $2,77(2,28-3,37)$ & $<0,0001$ & $1,37(1,10-1,72)$ & 0,0050 \\
Embarazo múltiple & $18,48(13,67-25,15)$ & $<0,0001$ & $15,56(11,04-21,93)$ & $<0,0001$ \\
Preeclampsia & $3,37(2,90-3,93)$ & $<0,0001$ & $2,40(1,62-3,57)$ & $<0,0001$ \\
Ruptura prematura de membranas & $1,24(1,04-1,48)$ & 0,0163 & $1,18(0,70-1,76)$ & 0,6630 \\
Corioamnionitis & $2,62(1,92-3,55)$ & $<0,0001$ & $0,91(0,44-1,88)$ & 0,7940 \\
Oligohidramnios & $4,18(3,12-5,56)$ & $<0,0001$ & $2,21(1,09-4,49)$ & 0,0270 \\
Hipertensión arterial crónica & $1,38(1,03-1,83)$ & 0,0194 & $1,14(0,55-2,37)$ & 0,7160 \\
Restricción del crecimiento intrauterino & $2,08(1,70-2,54)$ & $<0,0001$ & $1,91(1,09-3,32)$ & 0,0230 \\
Hemorragia del 3er trimestre (incluyendo DPP) & $16,18(9,96-26,80)$ & $<0,0001$ & $18,64(2,47-140,84)$ & 0,0050 \\
\hline
\end{tabular}

ORc: Odds ratio crudo. ORa: Odds ratio ajustado. CPN: Control prenatal. DPP: Desprendimiento prematuro de placenta. 


\section{DISCUSIÓN}

El presente trabajo se encargó de describir la prevalencia de prematuridad y sus determinantes de la salud, entre los nacimientos de una institución de salud colombiana.

Encontramos una prevalencia de prematuridad de $11,4 \%$, inferior a la informada por algunos países de América Latina como Costa Rica (13,6\%), El Salvador (12,8\%), Honduras $(12,2 \%)$, pero superior a Belice $(10,4 \%)$, Uruguay $(10,1 \%)$, Nicaragua $(9,3 \%)$, Brasil $(9,2 \%)$, Bolivia $(9 \%)$, Colombia $(8,8 \%)$, Panamá $(8,1 \%)$, Venezuela $(8,1 \%)$, Argentina $(8 \%)$, Paraguay $(7,8 \%)$, Guatemala $(7,7 \%)$, Perú $(7,3 \%)$, México $(7,3 \%)$, Chile $(7,1 \%)$, Cuba $6,4 \%)$ y Ecuador $(5,1 \%)(10)$. Nuestra prevalencia es más baja que la informada por Estados Unidos de Norteamérica (12,5\%) (9), pero superior a la informada en Europa (6,2\%) (17).

En cuanto al tipo de prematuros, encontramos que $89,3 \%$ fueron de $32-36$ semanas, $8,2 \%$ de $28-31$ semanas y $2,5 \%<28$ semanas. Esta distribución es similar a la descrita por otros autores, para quienes los extremadamente prematuros <28 semanas representan 5,2\% (IC 95\%: 5,1\%-5,3\%), muy prematuros $28-31$ semanas $10,4 \%$ (IC 95\%: $10,3 \%-10,5 \%$ ) y moderadamente prematuros $32-$ 36 semanas 84,3\% (IC 95\%: 84,1\%-84,5\%) (8,18).

Algunos de los determinantes maternos y gestacionales del parto prematuros de nuestros hallazgos, han sido descritos en la literatura. Algunas de estas causas condujeron a parto prematuro espontáneo, a inducción del trabajo de parto o al nacimiento por vía cesárea. El parto prematuro ocurre ya sea como consecuencia de un parto prematuro espontáneo, o después de la ruptura prematura de membranas ovulares (RPMO), o como consecuencia de un parto prematuro indicado por un proveedor de salud, ya sea como inducción del trabajo de parto o parto por cesárea antes de las 37 semanas de gestación completas por indicaciones maternas, obstétricas o fetales (19).

Un factor importante asociado al nacimiento prematuro es la poca adherencia al control prenatal (CPN). EI CPN adecuado se ha identificado como medida protectora contra el parto prematuro. Las últimas recomendaciones de organismos internacionales, como la OMS y National Institute for Health and Clinical Excellence $(20,21)$, dan más importancia a otros indicadores de calidad de la atención prenatal, que al número de visitas durante este, con al menos seis visitas considerado como óptimo. Esto surge del hecho de que no han encontrado diferencias estadísticamente significativas respecto a la morbilidad neonatal y materna, cuando se han completado más de seis visitas, siempre y cuando los demás indicadores de calidad se cumplan $(22,23)$.
El estudio realizado por Paz-Zuleta y cols (24), describe que el pobre cumplimiento de la atención prenatal es el principal factor de riesgo independiente asociado al parto prematuro y bajo peso al nacer. Los factores de riesgo social fueron importantes de manera independiente para el bajo peso al nacer.

Mendoza y cols (25), describen que la adolescencia, escolaridad $<11$ años, carencia de marido o compañero estable, carencia de seguridad social en salud y gravidez $\geq 2$, fueron los determinantes asociados a una mala adherencia al CPN. Esta mala adherencia al CPN se asoció significativamente a prematuridad y/o bajo peso al nacer y a mortalidad neonatal.

Redding y cols (26), evaluaron los resultados de un programa de acceso a salud comunitaria, utilizando agentes de salud comunitarios, para identificar a mujeres en riesgo de tener pobres resultados en el parto, conectarlos a los servicios de salud y servicios sociales para su atención y seguimiento. Encontraron una reducción del riesgo de nacimientos de bajo peso en el grupo intervenido por los agentes comunitarios $(6,1 \%$ vs $13 \%)$, convirtiéndola en una medida protectora.

La RPMO en embarazos simples, es responsable de $8,5-51,2 \%$ y el trabajo de parto espontáneo de $27,9 \%-65,4 \%$ de todos los partos prematuros. Para EUA, 69\% de los partos prematuros fueron espontáneos (27). Sin embargo, estos factores varían según la edad gestacional (28) y según factores sociales y ambientales, pero en la mayoría de los casos, las causas permanecen sin identificar (29). Varios factores han sido implicados, entre ellos el antecedente de parto prematuro previo (30), la edad de la madre (<20 años o >35 años), el periodo intergenésico corto y un índice de masa corporal bajo $(31,32)$.

Un factor importante asociado al parto prematuro es el embarazo múltiple, que aumenta el riesgo de parto prematuro en casi 10 veces, cuando se le compara con embarazos simples (33). El embarazo múltiple varía entre los grupos étnicos (1 de cada 40 mujeres de África Occidental, a 1 en 200 mujeres en Japón), aumenta a medida que la gestante es de mayor edad e incrementa con el aumento de la concepción asistida (34).

Las patologías infecciosas en la madre, juegan un papel importante en la ocurrencia del parto prematuro, incluyendo, infección del tracto urinario, vaginosis bacteriana, sífilis, infección por $\mathrm{VIH}$, corioamnionitis, colonización materna por Strepcococcus del grupo $B$, entre otros (35-37).

La insuficiencia de cuello cervical se ha asociado a infección uterina ascendente. (38). Otros factores asociados con el aumento de riesgo de parto prematuro son el tabaquismo y consumo de alcohol en exceso, así como la enfermedad periodontal 
(38), no evaluados en nuestro trabajo. La enfermedad periodontal, en especial la periodontitis apical crónica, se ha encontrado asociada al parto prematuro de muy bajo peso (39). Las anomalías congénitas son otra causa importante de parto prematuro espontáneo, mientras la prematuridad ocurre con mayor frecuencia en varones (40).

Hallamos también determinantes sociales, culturales y educativos, entre los nacimientos prematuros. Algunas de estas mujeres pertenecen a sectores de la sociedad muy desfavorecidos y con mayores inequidades en materia de salud, reflejado por ejemplo en una atención prenatal inadecuada (41). Son varias las categorías de determinantes que afectan la salud, algunos de ellos influenciados por la posición social (estado financiero, marginalidad social, desempleo a largo plazo, entorno físico, entorno laboral, etc.) (41-44). Deficiencia de servicios de salud en sectores de difícil acceso geográfico, como áreas rurales, donde hay ausencia de profesionales de la salud e insuficiente suministro continuo de insumos, incluyendo medicamentos $(41,42,46)$.

Nosotros no encontramos que la ruralidad fuera más frecuente entre los nacidos prematuramente. Se discute el papel que juega la etnicidad, pero esta se ve influenciada por factores socioeconómicos y estilos de vida, así como por la genética (45). El desplazamiento y la migración afectan principalmente a comunidades en situaciones socioeconómicas pobres y de violencia, y son estas poblaciones donde existe un mayor riesgo de inequidades entre los diferentes determinantes sociales, lo cual coloca a las mujeres, principalmente a las gestantes, en posiciones de riesgo social y vulnerabilidad (47-50), afectando los resultados neonatales (51-53).

La adolescencia es una época de la vida donde algunos de estos factores son más prevalentes, como lo encontramos en este trabajo. Entre las <20 años de edad, hay menor escolaridad, mayor carencia de seguridad social en salud y de esposo entre las variables sociales, culturales y educativas. Además en este grupo, hubo menor número de CPN, un período intergenésico más corto y corioamnionitis. La adolescencia se convierte en uno de los determinantes de una mala adherencia al CPN y esta a su vez a parto prematuro como fue descrito por Mendoza y cols (25), para quienes una edad $<20$ años aumenta en 85\% (IC 95\%: 44-139\%) el riesgo de no adherir al CPN y esta mala adherencia aumenta el riesgo de un parto prematuro en $66 \%$ (IC 95\%: 33-106\%). De otro lado, la anticoncepción en la adolescencia, principalmente, después de tener un hijo, es importante para alargar el periodo intergenésico que se asocia a prematuridad. El acceso a la anticoncepción principalmente entre la población adolescente, se ha identificado como una de las medidas protectoras contra el parto prematuro. Rodriguez y cols (54), evaluaron el impacto de la cobertura y uso de anticonceptivos dentro de los 18 meses posteriores a un nacimiento en la prevención del parto prematuro. Estos autores encontraron que, dentro de la cohorte evaluada, 9,75\% de partos fueron prematuros. Identificaron que la cobertura de anticonceptivos resultó ser protectora contra los nacimientos antes de la semana 37. Por cada mes de cobertura de anticonceptivos, las probabilidades de un parto prematuro disminuyeron 1,1\% (OR 0,989; IC 95\%: 0,986-0,993).

Finalmente, después del análisis multivariado, encontramos que los factores asociados al nacimiento prematuro incluyeron la etnia (indígena, afrocolombiano y mulato), un número de controles prenatales entre 0-6, un período intergenésico menor a 24 meses, el embarazo múltiple, la preeclampsia, corioamnionitis, oligohidramnios, la restricción de crecimiento intrauterino y las hemorragias del tercer trimestre (incluyendo desprendimiento prematuro de placenta).

\section{CONCLUSIONES}

La prematuridad es el resultado de una compleja red de determinantes individuales, sociales, culturales y gestacionales que interactúan. Es por ello, que para la prevención del parto prematuro, se debe trabajar no sólo en medidas de salud, sino en un sin número de medidas, que incluyen la elaboración de políticas y planes de acción integral que permitan minimizar las inequidades en el sector salud y en la atención de las gestantes. Dado que la prematuridad está íntimamente asociado a la calidad del CPN, a la planificación familiar entre los grupos vulnerables que incluyen a las adolescentes, se deben realizar intervenciones dirigidas a las mujeres en edad fértil, atendiendo sus necesidades en salud sexual y reproductiva, incluyendo educación sexual, uso de anticonceptivos, prevención de embarazos en la adolescencia temprana, prevención de embarazos no deseados, atención prenatal de calidad, cumplimiento del enfoque de riesgo para las gestantes e inclusión de personal calificado en la atención del parto. Si queremos reducir la morbilidad y la mortalidad neonatal e infantil, se deben emprender estrategias que permitan prevenir y tratar adecuadamente el nacimiento de prematuros.

\section{REFERENCIAS}

1. Garg A, Dworkin PH. surveillance and screening for social determinants of healththe medical home and beyond. JAMA Pediatr 2016;170(3):189-90.

2. Maness SB, Buhi ER. Associations between social determinants of health and pregnancy among young people. Public Health Reports 2016; 131(1): 86-99.

3 . International classification of diseases and related health problems. 10th revision. Geneva: World Health Organization; 1992. 
4. Huddy CL, Johnson A, Hope PL. Educational and behavioral problems in babies of 32-35 weeks gestation. Arch Dis Child Fetal Neonatal Ed 2001;85:F23F28.

5. Wang ML, Dorer DJ, Fleming MP, Catlin EA. Clinical outcomes of near-term infants. Pediatrics 2004;114:372-6.

6. Petrou $\mathrm{S}$. The economic consequences of preterm birth during the first 10 years of life. BJOG 2005;112:105.

7. Petrou S, Mehta Z, Hockley C, Cook-Mozaffari P, Henderson $\mathrm{J}$ and Goldacre $\mathrm{M}$. The impact of preterm birth on hospital inpatient admissions and costs during the first 5 years of life. Pediatrics 2003;112:1290-7.

8. March of Dimes, PMNCH, Save the Children, WHO. Born Too Soon: The Global Action Report on Preterm Birth. Eds CP Howson, MV Kinney, JE Lawn. World Health Organization. Geneva, 2012.

9. Martin JA, Hamilton BE, Menacker F, Sutton PD, Mathews TJ. Preliminary births for 2004: Infant and maternal health. Health e-stats. Released November 15, 2005. Disponible en: http://www.cdc.gov/nchs/ products/pubs/pubd/hestats/prelimbirths04/prelimbirths04health.htm. Acceso: 27-01-2016.

10. Blencowe $\mathrm{H}$, Cousens S, Oestergaard MZ, Chou D, Moller AB, Narwal R, et al. Nacido Demasiado Pronto: Informe de Acción Global sobre Nacimientos Prematuros. Datos de estimaciones nacionales, regionales y mundiales de la tasa de nacimientos prematuros en el año 2010. Disponible en: www.who.int/pmnch/media/ news/2012/preterm_birth_report/es/index.html. Acceso: 28-01-2016.

11. Lawn JE, Wilczynska-Ketende K, Cousens SN. Estimating the causes of 4 million neonatal deaths in the year 2000. Int J Epidemiol 2006;35:706-18.

12. Liu L, Johnson H, Cousens S, Perin J, Scott S, Lawn $\mathrm{J}$, Ruden I, et al. Global, regional and national causes of child mortality: an updated systematic analysis for 2010 with time trends since 2000. Lancet 2012;379:2151-61.

13. Goldenberg RL, Jobe AH. Prospects for research in reproductive health and birth outcomes. JAMA 2001;285:633-9.

14. Kramer MS, Demissie K, Yang H, Platt RW, Sauve R, Liston $\mathrm{R}$. The contribution of mild and moderate preterm birth to infant mortality. Fetal and Infant Health Study Group of the Canadian Perinatal Surveillance System. JAMA 2000;284: 843-9.

15. Lawn JE, Kerber K, Enweronu-Laryea C, Cousens S. 3.6 million neonatal deaths - what is progressing and what is not? Semin Perinatol 2010;34:371-86.

16. Slattery MM, Morrison JJ. Preterm delivery. Lancet 2002;360:1489-97.

17. Beck S, Wojdyla D, Say L, Betran AP, Merialdi M, Requejo $\mathrm{JH}$, et al. The worldwide incidence of preterm birth: a systematic review of maternal mortality and morbidity. Bull World Health Organ 2010;88:31-8.

18. Blencowe $H$, Cousens S, Chou D, Oestergaard $M$, Say L, Moller A-B, et al. Born Too Soon: The global epidemiology of 15 million preterm births. Reprod Health 2013: 10 Suppl 1:S2.

19. Goldenberg RL, Gravett MG, lams J, Papageorghiou AT, Waller SA, Kramer M, et al. The preterm birth syndrome: issues to consider in creating a classification system. Am J Obstet Gynecol 2012;206:113-8.
20. World Health Organization. Department of Reproductive Health and Research. Randomized Trial WHO antenatal care: a manual for the implementation of the new antenatal care model; 2003. Disponible en: http:// whqlibdoc.who.int/hq/2001/WHO_RHR_01.30.pdf. Acceso: 24-03-2016.

21. Antenatal care routine care for healthy pregnant woman. National Collaborating Centre for Women's and Children's Health. National Institute for Health and Clinical Excellence. NICE Clinical Guideline 62; 2008. Disponible: http://www.nice.org.uk/nicemedia/pdf/ CG62fullguideline.pdf. Acceso: 24-03-2016.

22. Villar J, Carroli G, Khan-Neelofur D, Piaggio G, Gulmezoglu M. Patterns of routine antenatal care for low-risk pregnancy. Cochrane Database Syst Rev. 2001;(4)(4):CD000934.

23. Villar J, Ba'aqeel $H$, Piaggio $G$, Lumbiganon $P$, Miguel Belizan J, Farnot U, et al. WHO antenatal care randomized trial for the evaluation of a new model of routine antenatal care. Lancet 2001;357: 1551-64.

24. Paz-Zulueta M, Llorca J, Sarabia-Lavín R, Bolumar F, Rioja L, Delgado A, et al. The Role of Prenatal Care and Social Risk Factors in the Relationship between Immigrant Status and Neonatal Morbidity: A Retrospective Cohort Study. PLoS One 2015;10(3):e0120765.

25. Mendoza LA, Arias MD, Peñaranda CB, Mendoza LI, Manzano S, Varela AM. Influencia de la adolescencia y su entorno en la adherencia al control prenatal e impacto sobre la prematuridad, bajo peso al nacer y mortalidad neonatal. Rev Chil Obstet Ginecol 2015;80(4):306-15.

26. Redding S, Conrey E, Porter K, Paulson J, Hughes K, Redding M. Pathways Community Care Coordination in Low Birth Weight Prevention. Maternal Child Health J 2015;19(3):643-50.

27. Ananth CV, Joseph KS, Oyelese Y, Demissie K and Vintzileos AM. Trends in preterm birth and perinatal mortality among singletons: United States, 1989 through 2000. Obstet Gynecol 2005;105:1084-91.

28. Steer P. The epidemiology of preterm labour. BJOG 2005;112(Suppl 1):1-3.

29. Menon R. Spontaneous preterm birth, a clinical dile$\mathrm{mma}$ : etiologic, pathophysiologic and genetic heterogeneities and racial disparity. Acta Obstet Gynecol Scand 2008:87:590-600.

30. Plunkett J, Muglia LJ. Genetic contributions to preterm birth: implications from epidemiological and genetic association studies. Ann Med 2008;40:167-95.

31. Goldenberg RL, Culhane JF, lams JD, Romero R. Epidemiology and causes of preterm birth. Lancet 2008;371:75-84.

32. Muglia LJ, Katz M. The enigma of spontaneous preterm birth. N Engl J Med 2010;362:529-35.

33. Blondel B, Macfarlane A, Gissler M, Breart G, Zeitlin J. Preterm birth and multiple pregnancy in European countries participating in the PERISTAT project. BJOG 2006;113:528-35.

34. Felberbaum RE. Multiple pregnancies after assisted reproduction - international comparison. Reprod Biomed Online 2007;15(Suppl 3):53-60.

35. Simonsen KA, Anderson-Berry AL, Delair ShF, Davies HD. Early-Onset Neonatal Sepsis. Clin Microbiol Rev 2014;27(1):21-47.

36. Chan GJ, Lee A, Baqui AH, Tan J, Black RE. Prevalence of early-onset neonatal infection among 
newborns of mothers with bacterial infection or colonization: a systematic review and meta-analysis. BMC Infectious Diseases 2015;15:118.

37. Polin RA; Committee on Fetus and Newborn. Management of neonates with suspected or proven earlyonset bacterial sepsis. Pediatrics 2012;129(5):100615.

38. Lee SE, Romero R, Park CW, Jun JK, Yoon BH. The frequency and significance of intraamniotic inflammation in patients with cervical insufficiency. Am J Obstet Gynecol 2008;198(6):633.e1-8.

39. Mendonca AS, Figueiredo AE, Oliveira LM, Ferreira F, Pinheiro V, Fróes K, et al. Association between chronic apical periodontitis and low-birth-weight preterm births. J Endodontics 2015;41(3):353-7.

40. Zeitlin J, Saurel-Cubizolles MJ, De Mouzon J, Rivera L, Ancel PY, Blondel B, Kaminski M. Fetal sex and preterm birth: are males at greater risk? Hum Reprod 2002;17:2762-8.

41. World Health Organization. State of Inequality: Reproductive, Maternal, Newborn and Child Health. Geneva: WHO Press; 2015.

42. Diderichsen F, Andersen I, Manuel C; Working Group of Danish Review on Social Determinants of Health, Andersen AM, Bach E, Baadsgaard M, BrønnumHansen $H$, Hansen FK, Jeune B, Jørgensen T, Søgaard J. Health inequality - Determinants and policies. Scand J Public Health 2012;40(8 Suppl): 12105.

43. Chen L, Wu Y, Coyte PC. Income related children's health inequality and health achievement in China. Int J Equity Health 2014;13:102.

44. Ataguba JE, Akazili J, McIntyre D. Socioeconomic related health inequality in South Africa: Evidence from General Household Surveys. Int J Equity Health 2011;10:48.

45. Ananth CV, Vintzileos AM. Epidemiology of preterm birth and its clinical subtypes. J Matern Fetal Neonatal Med 2006;19:773-82.
46. Park K, editor. Health care of the community. In: Textbook of Preventive and Social Medicine. 20th ed. Jabalpur: Banarsidas Bhanot Publishers; 2009. Pp 793 4.

47. Reijneveld SA. Reported health lifestyles and use of health care of first generation immigrants in The Netherlands: do socioeconomic factors explain their adverse position? J Epidemiol Community Health 1998;52:298-304.

48. Dunn JR, Dyck I. Social determinants of health in Canada's immigrant population: results from the National Population Health Survey. Soc Sci Med 2000;51: 1573-93.

49. Borrell C, Muntaner C, Sola J, Artazcoz L, Puigpinos R, Benach J, et al. Immigration and self-reported health status by social class and gender: the importance of material deprivation, work organization and household labour. J Epidemiol Community Health 2008;62(5)e7.

50. Malmusi D, Borrell C, Benach J. Migration-related health inequalities: showing the complex interactions between gender, social class and place of origin. Soc Sci Med 2010;71:1610-9.

51. Rodriguez C, Regidor E and Gutierrez-Fisac JL. Low birth weight in Spain associated with sociodemographic factors. J Epidemiol Community Health 1995;49:38-42.

52. Sparks PJ. Do biological, sociodemographic, and behavioral characteristics explain racial/ethnic disparities in preterm births? Soc Sci Med. 2009;68:1667-75.

53. Garcia-Subirats I, Perez G, Rodriguez-Sanz M, Salvador J, Jane M. Recent immigration and adverse pregnancy outcomes in an urban setting in Spain. Matern Child Health J 2011;15:561-9.

54. Rodriguez MI, Chang R, Thiel de Bocanegra $\mathrm{H}$. The impact of postpartum contraception on reducing preterm birth: findings from California. Am J Obstet Gynecol 2015;213:703.e1-6. 